\title{
Stability of single particle motion with head-on beam- beam compensation in the RHIC
}

\author{
Y. Luo, W. Fischer, N. Abreu
}

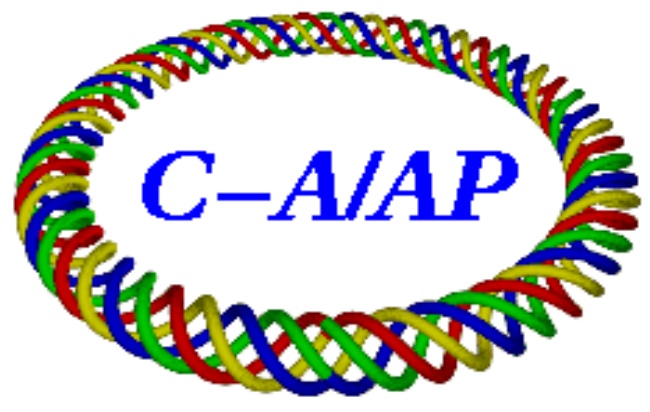

Collider-Accelerator Department Brookhaven National Laboratory Upton, NY 11973

Notice: This document has been authorized by employees of Brookhaven Science Associates, LLC under Contract No. DE-AC02-98CH10886 with the U.S. Department of Energy. The United States Government retains a non-exclusive, paidup, irrevocable, world-wide license to publish or reproduce the published form of this document, or allow others to do so, for United States Government purposes. 
May 6, 2008

\title{
Stability of single particle motion with head-on beam-beam compensation in the RHIC
}

\author{
Y. Luo, W. Fischer and N. Abreu \\ Brookhaven National Laboratory, Upton, NY 11973, USA
}

To compensate the large tune shift and tune spread generated by the head-on beam-beam interactions in the polarized proton run in the Relativistic Heavy Ion Collider (RHIC), we proposed a low energy electron beam with a Gaussian transverse profiles to collide head-on with the proton beam. In this article, with a weak-strong beam-beam interaction model, we investigate the stability of single particle motion in the presence of head-on beam-beam compensation. Tune footprints, tune diffusion, Lyapunov exponents, and $10^{6}$ turn dynamic apertures are calculated and compared between the cases without and with beam-beam compensation. A tune scan is performed and the possibility of increasing the bunch intensity is studied. The cause of tune footprint foldings is discussed, and the tune diffusion and Lyapunov exponent analysis are compared.

\section{Introduction}

The current RHIC working points for the proton beams are constrained between $2 / 3$ and $7 / 10$ [1]. It has been shown by both experiments and simulations that when the fractional betatron tune is close to $2 / 3$, the beam lifetime will be affected by the strong third order betatron resonance. And when the vertical tune is close to $7 / 10$ both the luminosity lifetime and proton polarization will be negatively affected. The nominal working points without collisions are currently $(28.685,29.695)$ and $(28.695,29.685)$ for the two RHIC rings. In the 2008 run the proton bunch intensity had reached about $1.7 \times 10^{11}$ in the Blue ring. To further increase the bunch intensity to $2.0 \times 10^{11}$ or even higher, there may not be enough tune space between $2 / 3$ and $7 / 10$ resonances.

One possible solution is to adopt head-on beam-beam compensation $[2,3]$. In the Tevatron at Fermilab, a low energy electron beam, called electron lens or e-lens, has been used to compensate the tune shift of PACMAC bunches. The tune shift of these bunches is caused by long-range beam-beam interactions [4]. Experimentally the tune shift compensation increases the lifetime of the PACMAN bunches [5]. In our study, we investigate whether a device like the Tevatron e-lens can be used to mitigate the head-on beam-beam effects in RHIC.

To evaluate the benefits and side effects of the head-on beam-beam compensation with an e-lens in RHIC, simulation studies were done. In 2005 a preliminary study showed that e-lenses in the RHIC rings reduce the tune shift and tune spread generated by the head-on beam-beam interactions. The studies were extended later to also evaluate lifetime and emittance evolution $[6,7]$.

In this article, we report on studies of the single particle motion stability in the presence of head-on beam-beam compensation in RHIC. We will introduce the parameters of the proton and electron beams, the lattice for this study, the beam-beam interaction model, and the tracking code. Then, we calculate and compare, for cases without and with head-on beam-beam compensation, tune footprints, tune diffusion and Lyapunov exponents from short term tracking, and dynamic apertures up to $10^{6}$ turn. We test several tunes and evaluate the possibility of increasing the bunch intensity. The cause of the tune footprint foldings is discussed, and tune diffusion and Lyapunov exponent analysis are compared.

\section{Beam parameters and weak-strong beam-beam model}

For the RHIC pp run, the two proton beams collide at IP6 and IP8. The proton beam in the Blue ring circulates clockwise, while the proton beam in the Yellow ring circulates anti-clockwise. In the current design of RHIC head-on beam-beam compensation, e-lenses are located close to IP10. Figure 1 gives the layout of the RHIC head-on beam-beam compensation. 
Two e-lenses are needed for the RHIC head-on beam-beam compensation, one for the Blue ring and another one for the Yellow ring. The two proton beams are vertically separated at the IP10. The e-lens for the Blue ring is named BEL, and the e-lens for the Yellow ring is named YEL. They are assumed to be $2 \mathrm{~m}$ long. They are symmetrically placed 1.5 meter away from IP10. However, in the following simulations we assume, for simplicity, the e-lenses are exactly located at IP10.

Tab. 1 lists the proton beam parameters in this study. The proton energy is $250 \mathrm{GeV}$, corresponding to a relativistic factor $\gamma=266$. The beta functions at IP6 and IP10 are $\beta_{x, y}^{*}=0.5 \mathrm{~m}$. The beta functions at IP10, where the e-lenses are located, are $\beta_{x, y}^{e}=10 \mathrm{~m}$. The beta functions at other interaction points (IP2, IP4, IP10) are $10 \mathrm{~m}$. In the simulation study, two nominal (i.e. without collision) working points are used: $(28.695,29.685)$ and $(28.685,29.695)$. The linear chromaticities are set to $\xi_{x, y}=+1$. The second order chromaticities are not corrected in this study. The multipole magnetic field errors in the triplet quadrupoles and separation dipole magnets in the interaction regions (IRs) are included in the lattice model.

The bunch intensity is chosen as $N_{p}=2.0 \times 10^{11}$. The proton beam rms transverse emittance is assumed to be $2.5 \mathrm{~mm} \cdot \mathrm{mrad}$ ( $15 \mathrm{~mm} \cdot \mathrm{mrad}$ for the $95 \%$ emittance). The normalized rms longitudinal bunch area of the proton beam is assumed to be $0.17 \mathrm{eV} \cdot \mathrm{s}$. The relative $\mathrm{rms}$ momentum spread of the proton beam is $\delta_{r m s}=\left(\frac{\Delta p}{p_{0}}\right)_{r m s}=0.14 \times 10^{-3}$, the rms bunch length of the proton beam is $\sigma_{l}=0.44 \mathrm{~m}$.

For the best head-on beam-beam compensation, in this study we assume that the electron beams have the same transverse Gaussian profiles as the proton beams at IP10. For the full head-on beam-beam compensation, the electron particle density is twice of that of proton bunch intensity, that is $N_{e}=4.0 \times 10^{11}$. For half head-on beam-beam compensation, the electron particle intensity is $N_{e}=2.0 \times 10^{11}$. Full and half head-on beam-beam compensations will compensate full and half of the linear beam-beam tune shifts respectively.

In our study the 6-D simplectic tracking code SixTrack [8] is used. In this code, the linear elements are treated as thick elements while the nonlinear elements are treated as thin-lenses. The beam-beam interaction is based on a weak-strong model. For simplicity and calculational speed a 4-D beam-beam kick á la Basetti and Erskine [9] is used. The beam-beam kicks from the electron beam on the proton particles are applied at IP10.

As a convention, we always launch initial particles for tracking in the first quadrant of the $\left(x / \sigma_{x}, y / \sigma_{y}\right)$ plane. The initial conjugate momenta are set zero, $p_{x}=p_{y}=0$. The initial coordinates of particles are sampled uniformly between $0^{\circ}$ to $90^{\circ}$ in the $\left(x / \sigma_{x}, y / \sigma_{y}\right)$ plane. In the calculations of tune footprints, tune diffusion and Lyapunov exponents, the initial coordinates of particles are sampled uniformly from 0 to $6 \sigma$. In our dynamic aperture calculation, fast binary searching is adopted.

In the following study, we calculate 4-D tune footprints, 4-D tune diffusion and 6-D Lyapunov exponents over 2048 turns to estimate the long-term stability of single particle motion. Limited by the available CPU time and computational rounding errors, dynamic apertures are calculated through direct tracking up to $10^{6}$ turns.

\section{Tune footprint calculations in 4-D tracking}

The initial motivation of adopting an e-lens for RHIC is to compensate the head-on beam-beam interaction generated tune shift and tune spread. The tune shift for particles with small betatron amplitudes from beam-beam interaction at one IP is

$$
\xi=-\frac{N_{p} r_{0}}{4 \pi \epsilon_{n}} .
$$

Here $N_{p}$ is the number of protons per bunch, $r_{0}$ is the classical proton radius, $\epsilon_{n}$ is the normalized rms transverse emittance, and $\xi$ is called the beam-beam parameter.

The nominal tunes without collision are $(28.685,29.695)$ and $(28.695,29.685)$. Assuming a transverse normalized $\mathrm{rms}$ emittance of $\epsilon_{n}=2.5 \mathrm{~mm} \cdot \mathrm{mrad}$, the total beam-beam tune shift from two collisions at IP6 and IP8 with $N_{p}=2.0 \times 10^{11}$ is about -0.02 . Therefore, with $N_{p}=2.0 \times 10^{11}$, the beam-beam tune shift will push the beam with nominal working point $(28.685,29.695)$ onto the horizontal third order resonance line and push the beam with nominal working point $(28.695,29.685)$ onto the vertical third order resonance line.

In this section, we calculate the tune footprints without and with beam-beam compensation. Initial particles are launched in the $\left(x / \sigma_{x}, y / \sigma_{y}\right)$ plane from 0 to $6 \sigma$ with a step size $0.1 \sigma$ and from $5^{\circ}$ to $85^{\circ}$ with a step size $5^{\circ}$. Each particle is tracked for 2048 turns. The betatron tunes are calculated with Sussix [10].

The top-left plot in Fig. 2 shows the footprints of on-momentum particles without and with headon beam-beam interactions for both nominal working points. The footprints above the diagonal are for 
Table 1: Parameters for the proton beams

\begin{tabular}{lcc}
\hline \hline quantity & unit & value \\
\hline lattice & $\mathrm{m}$ & 3833.8451 \\
ring circumference & $\mathrm{GeV}$ & 250 \\
energy & - & 266 \\
relativistic $\gamma$ & - & $\mathrm{IP} 6, \mathrm{IP} 8$ \\
beam-beam collision points & - & $\mathrm{IP} 10$ \\
beam-beam compensation point & $\mathrm{m}$ & 0.5 \\
$\beta_{x, y}^{*}$ at IP6 and IP8 & $\mathrm{m}$ & 10 \\
$\beta_{x, y}^{e}$ at IP10 & $\mathrm{m}$ & 10 \\
$\beta_{x, y}^{*}$ at all other IPs & & \\
\hline proton beam & - & $2 \times 10^{11}$ \\
particles per bunch $N_{p}$ & $\mathrm{~mm} \cdot \mathrm{mrad}$ & 2.5 \\
normalized transverse rms emittance $\epsilon_{x, y}$ & $\mathrm{~mm}$ & 0.068 \\
transverse rms beam size at collision points $\sigma_{x, y}^{*}$ & $\mathrm{~mm}$ & 0.31 \\
transverse rms beam size at e-lens $\sigma_{x, y}^{e}$ & - & $(28.695,29.685)$ \\
transverse tunes $\left(Q_{x}, Q_{y}\right)$ & - & $(1,1)$ \\
chromaticities $\left(\xi_{x}, \xi_{y}\right)$ & - & -0.01 \\
beam-beam parameter per IP $\xi_{\mathrm{p} \rightarrow \mathrm{p}}$ & & \\
\hline longitudinal parameters & - & 360 \\
harmonic number & $\mathrm{kV}$ & 300 \\
rf cavity voltage & $\mathrm{eV} \cdot \mathrm{s}$ & 0.17 \\
rms longitudinal bunch area & - & $0.14 \times 10^{-3}$ \\
rms momentum spread & $\mathrm{m}$ & 0.44 \\
rms bunch length & & \\
\hline \hline
\end{tabular}

nominal working point $(28.685,29.695)$ and the footprints below diagonal are for nominal working point $(28.695,29.685)$. Different colors in Fig. 2 show different initial particle amplitudes. From the top-left plot of Fig. 2, with beam-beam interactions at IP6 and IP8, the particles in the bunch core of the beam with nominal working point $(28.685,29.695)$ are pushed onto the horizontal third order resonances, while the particles in the bunch core of the beam with nominal working point $(28.695,29.685)$ are pushed onto the vertical third order resonances. The beam-beam interactions also generate a much larger tune spread than the nonlinear magnetic fields.

The top-right plot in Fig. 2 shows the footprints of on-momentum particles with half and full head-on beam-beam compensations for both nominal working points. With head-on beam-beam compensation, the tune spreads generated by the proton-proton interactions are also greatly reduced. The particles in the bunch cores are pulled away from the third order betatron resonance lines. With full head-on beam-beam compensations, the final tune spreads are comparable to those from magnetic nonlinear fields only.

In the top-right plot of Fig. 2 foldings in the tune footprints [11] in radial and azimuthal directions are visible, especially for the case with full beam-beam compensations. Without beam-beam interaction, there is no tune footprint folding up to $6 \sigma$. With beam-beam interactions at IP6 and IP8 only, the tune footprint folding occur around $5 \sigma$. With half beam-beam compensation, it happens at $4 \sigma$. With full beam-beam compensation, the tune footprint foldings start at very small amplitudes and the folding occur in both radial and azimuthal directions.

The two bottom plots in Fig. 2 show the tune footprints for the off-momentum particles. The relative momentum deviation is $\Delta p / p_{0}=0.0005$ which is about $3 \delta_{p}$. In our calculation, the first order chromaticities are set to $\xi_{x, y}=+1$ and the second order chromaticities are not corrected. There is no significant difference in the shapes of tune footprints between on- and off-momentum particles.

In this section we verified that a head-on beam-beam compensation can greatly reduce the beam-beam generated large tune shift and tune spread. However, with head-on beam-beam compensation, tune footprint foldings occur at amplitudes lower than for the case without beam-beam compensation. Tune footprint foldings will be discussed further below. 


\section{Tune diffusion calculation in 4-D tracking}

In this section we calculate and compare 4-D tune diffusion for on-momentum particles without and with beam-beam compensation. Tune diffusion analysis, more generally called frequency map analysis, has been used for the study of single particle stability since J. Laskar introduced it into Accelerator Physics from the study of the solar system in 1990s [14, 15]. Small tune diffusion from short-term tracking is used as an indicator of long-term particle stability. We calculate the tune diffusion in 4-D tracking.

In our study the initial particle coordinates are sampled uniformly from 0 to $6 \sigma$ with step size $0.1 \sigma$ and from $1^{\circ}$ to $89^{\circ}$ with step size $1^{\circ}$ in the $\left(x / \sigma_{x}, y / \sigma_{y}\right)$ plane. The initial conjugate momenta are set to zero, that is $p_{x}=p_{y}=0$. Each particle is tracked to 2048 turns. The betatron tunes are calculated in the first and second 1024 turns. A measure for the tune diffusion over 2048 turns is the tune change

$$
|\Delta Q|=\sqrt{\left|\Delta Q_{x}\right|^{2}+\left|\Delta Q_{y}\right|^{2}},
$$

where $\left|\Delta Q_{x}\right|$ and $\left|\Delta Q_{y}\right|$ are the horizontal and vertical betatron tune differences between the first and second 1024 turns. The tunes are determined with Sussix.

The tune diffusion can be shown in both the $\left(x / \sigma_{x}, y / \sigma_{y}\right)$ and the $\left(Q_{x}, Q_{y}\right)$ plane where different colors show different tune change values. Deep and light blue dots mean that the particles they represent have tune changes of less than $10^{-5}$. Black dots denote particles that have tune changes larger than $10^{-2}$ which are likely much less stable. The particles represented by the green and yellow dots have tune changes between those denoted by blue and black.

Figure 3 and Fig. 4 show the tune changes of on-momentum particles for nominal working points (28.685, $29.695)$ and $(28.695,29.685)$ in the $\left(x / \sigma_{x}, y / \sigma_{y}\right)$ plane respectively. In both figures, the top-left and topright plots show the tune changes for the cases without and with beam-beam interactions respectively. The bottom-left and bottom-right plots show the tune changes for the cases with half and full head-on beam-beam compensation respectively.

From Fig. 3 and Fig. 4, particles without beam-beam interactions below $6 \sigma$ have tune changes smaller than $10^{-4}$. There is a large continuous deep and light blue area from 0 up to $4 \sigma$. With beam-beam interactions the large continuous blue area disappears. And several yellow curves with tune changes from $10^{-4}$ to $10^{-3}$ appear from 0 to $4-5 \sigma$. Beyond $4-5 \sigma$, there is an area scattered by some small yellow spots.

From Fig. 3 and Fig. 4 we see that with head-on beam-beam compensations the tune diffusion for particles below $3 \sigma$ is reduced compared to the case without compensation. A small continuous blue area appears from 0 up to $2-3 \sigma$. With half beam-beam compensation one or two green curves instead of yellow curves are visible below $3 \sigma$. With full beam-beam compensation the area above $4 \sigma$ showing mixed colors (black, yellow, and green) expands. More black dots are seen for the cases with beam-beam compensation.

The yellow and green curves in Fig. 3 and Fig. 4 are linked to resonances. In Fig. 5 we plot the same tune changes in the $\left(Q_{x}, Q_{y}\right)$ plane. Zooming into the tune footprints with half beam-beam compensation in Fig. 5, the same resonances are visible before and after tune footprint foldings. Before the foldings the resonance line is colored in green with tune changes between $10^{-5}$ to $10^{-4}$. After foldings, the same resonance line turns to black with tune changes larger than $10^{-2}$. This is an evidence that foldings in the tune footprint should be avoided.

In Fig. 5 with beam-beam interactions, resonances are visible at low amplitudes. With full beam-beam compensation, crossings of yellow resonance lines are visible beginning at $4 \sigma$. These resonance crossings produce the color-mixed area in Fig. 3 and Fig. 4. According to Refs. [12, 13], resonance crossing in the $\left(Q_{x}, Q_{y}\right)$ plane, or resonance overlapping in $\left(x-p_{x}\right),\left(y-p_{y}\right)$ phase spaces indicate chaotic motion.

We conclude from the tune diffusion analysis that head-on beam-beam compensations will reduce tune diffusion of particles below $3 \sigma$. However resonance crossing or resonance overlapping occurs at smaller betatron amplitudes.

\section{Lyapunov exponent calculation in 6-D tracking}

The Lyapunov exponent is another indicator of long-term stability of single particle motion. Two particles are launched with a small distance in phase space. Their distance will grow linearly for regular motion or exponentially for chaotic motion. We define the Lyapunov exponent after $n$ turns as [16]

$$
\lambda(n)=\frac{1}{n} \ln \frac{\left|\mathbf{X}_{\mathbf{2}}(n)-\mathbf{X}_{\mathbf{1}}(0)\right|}{d_{0}}
$$


$\mathbf{X}_{\mathbf{i}}(n), \mathrm{i}=1,2$, are the coordinate vectors of the two particles after turn $n$. The initial distance of these two particles in phase space is $\left|\mathbf{X}_{\mathbf{2}}(0)-\mathbf{X}_{\mathbf{1}}(0)\right|=d_{0}$. $d_{0}$ should be very small to make the analysis meaningful. If the particle motion is regular, the distance will grow linearly, and $\lambda(n)$ tends to zero. If the motion is chaotic, the distance of these two particles grows exponentially and $\lambda(n)$ converges to a positive value.

In SixTrack, these two particles are called twin particles. The difference in the twin particles' initial coordinates can be in any of the phase space dimensions $\left(x, p_{x}, y, p_{y}, c \Delta t, \delta_{p}\right)$. In our study, the coordinate of first particle is uniformly sampled in the $\left(x / \sigma_{x}, y / \sigma_{y}\right)$ plane with zero conjugate momenta $p_{x}=p_{y}=0$, from 0 to $6 \sigma$ with a step size of $0.1 \sigma$ and from $1^{\circ}$ to $89^{\circ}$ with a step size of $1^{\circ}$. The second particle's $x$ - and $y$-coordinates differs from that of the first particle by $0.707 \times 10^{-6} \mathrm{~mm}$. With that we have $d_{0}=10^{-6} \mathrm{~mm}$. Particles are tracked over 2048 turns, with longitudinal motion included. However, for simplicity, we calculate the Lyapunov exponents from the transverse planes only.

As an example, Fig. 6 shows the distances and the Lyapunov exponents for the regular and chaotic motions. The two left plots show the distances and the two right plots show the Lyapunov exponents as a function of the turn number $n$. The two top plots are for the regular motion. The two bottom plots are for chaotic motion. From Fig. 6 the distance of the twin particles for the regular motion growth linearly and steadily. Its Lyapunov exponent decreases monotonically in 2048 turns. For the chaotic motion, the distance of the twin particles grows much faster and nonlinearly. Two exponential growth periods are seen between 500-1200 turns and between 1600-2048 turns. In the following we will plot $\lambda(2048)$ in the $\left(x / \sigma_{x}, y / \sigma_{y}\right)$ plane, which we call the Lyapunov exponent map.

Figure 7 and Fig. 8 show the maximum Lyapunov exponents in the $\left(x / \sigma_{x}, y / \sigma_{y}\right)$ plane for working points $(28.685,29.695)$ and $(28.695,29.685)$ respectively. In Fig. 7 and Fig. 8 , the top-left and top-right plots show the maximum Lyapunov exponents without and with beam-beam interactions respectively. The bottom-left and bottom-right plots show the maximum Lyapunov exponents with half and full beam-beam compensations. In each plot different colors denote different ranges of $\lambda(2048)$. For example, the deep and light blue dots show the particles having $\lambda(2048)$ smaller than 0.001 . The black dots show the particles with $\lambda(2048)$ larger than 0.003 , which we expect to be less stable than particles denoted by blue dots.

From the top plots of Fig. 7 and Fig. 8, particles without beam-beam interactions below $3 \sigma$ are very stable. Pink dots are only seen beyond $5 \sigma$. There are no black dots up to $6 \sigma$. With beam-beam interactions, pink and red dots occupy the area from $1 \sigma$ to $4.5 \sigma$. Below $1 \sigma$ some red dots are visible. Beyond $4.5 \sigma$ the area is filled with dots with different colors. Resonance lines are visible in the Lyapunov exponent map.

From the bottom plots of Fig. 7 and Fig. 8, with half the head-on beam-beam compensation, the bunch core is more stable compared to the case without compensation. With half beam-beam compensation, the pink dots dominate the area from $2 \sigma$ to $3 \sigma$ in the $\left(x / \sigma_{x}, y / \sigma_{y}\right)$ plane. With full beam-beam compensations, pink and red dots are moved up from $3 \sigma$. Therefore, with half and full beam-beam compensation, the particles with small amplitudes are more stable than in the case of without compensation. However, note that with full beam-beam compensation, especially for the working point $(28.685,29.695)$, there are many more black dots above $4 \sigma$. These black dots gather on a wide resonance strip.

From the analysis of Lyapunov exponent maps, a similar conclusion can be drawn as that from tune diffusion analysis. The head-on beam-beam compensation stabilizes particles below $3 \sigma$ but destabilizes particles beyond $4 \sigma$.

\section{Dynamic aperture calculations in 6-D tracking}

In this section we calculate dynamic apertures in 6-D tracking without and with head-on beam-beam compensation. The dynamic aperture (DA) is defined as the maximum phase-space amplitude within which particles do not get lost after a certain number of turns [18]. The long-term dynamic aperture converges to the boundary between the regular and chaotic motions [16]. However, limited by available CPU time and rounding errors, direct particle tracking beyond $10^{7}$ is not practical.

In the following, we determine dynamic apertures in the $\left(x / \sigma_{x}, y / \sigma_{y}\right)$ plane with $10^{6}$ turn tracking. The initial particles are sampled from $5^{\circ}$ to $85^{\circ}$ with a step size of $5^{\circ}$, and from 0 to $12 \sigma$ with an amplitude step size of $0.2 \sigma$. The particle's initial conjugate momenta are set to zero, $p_{x}=p_{y}=0$. Longitudinal motion is included.

There are some differences in the dynamic apertures among the phase angles in the $\left(x / \sigma_{x}, y / \sigma_{y}\right)$ plane. To simplify the comparison, we focus on the minimum dynamic aperture $D_{\min }$ and the phase averaged 
dynamic aperture $D_{\text {avg }}$. The phase averaged dynamic aperture can be defined as [17]

$$
D_{\text {avg }}=\left(\sum_{i=1}^{9} D\left(\alpha_{i}\right)^{4} \sin \left(2 \alpha_{i}\right) \Delta \alpha\right)^{\frac{1}{4}} .
$$

$D\left(\alpha_{i}\right)$ is the dynamic aperture in phase space direction $\alpha_{i}, \Delta \alpha$ is angle step size.

Figure 9 shows the $10^{6}$ turn dynamic apertures for the same working point but with different beam-beam conditions. The top-left and top-right plots show the $10^{6}$ turn dynamic apertures for the nominal working point $(28.685,29.695)$. The bottom-left and bottom-right plots show the $10^{6}$ turn dynamic apertures for the nominal working point $(28.695,29.685)$. Alternatively Fig. 10 shows dynamic apertures with the same beam-beam condition but for different working points. The top-left and top-right plots show the dynamic apertures without and with beam-beam interaction respectively. The bottom-left and bottom-right plots show the dynamic apertures with half and full beam-beam compensations respectively.

Table 9 lists the $10^{6}$ turn dynamic apertures found in each phase angle and the minimum and phaseaveraged dynamic apertures among these 9 angles. According to Tab. 9, for the same beam-beam conditions, the dynamic apertures for on- and off-momentum particles are slightly different. The differences are less than $0.5 \sigma$. The relative momentum deviation for off-momentum particles is $\Delta p / p_{0}=0.0005$. In the following, we focus on comparing the dynamic apertures of off-momentum particles.

From Tab. 9, for off-momentum particles, the minimum and angle averaged dynamic apertures without beam-beam interaction are $7.6 \sigma$ and $8.5 \sigma$ for the working point $(28.685,29.695)$, and are $8.0 \sigma$ and $8.8 \sigma$ for the working point $(28.695,29.685)$. With beam-beam interactions at IP6 and IP8, the minimum and angle averaged dynamic apertures are reduced by about $2 \sigma$.

With head-on beam-beam compensations, for off-momentum particles, both minimum and angle averaged dynamic apertures are reduced, comparing to that without compensation. For working point (28.685, 29.695), the minimum and angle averaged dynamic apertures drop by $0.7 \sigma$ and $0.3 \sigma$ with half beam-beam compensation and drop by $1.1 \sigma$ and $0.7 \sigma$ with full beam-beam compensation respectively. For working point $(28.695,29.685)$, the minimum and angle averaged dynamic apertures drop by $0.3 \sigma$ and $0.1 \sigma$ with half beam-beam compensation and by $0.3 \sigma$ and $0.3 \sigma$ with full beam-beam compensations. Therefore, with head-on beam-beam compensation, we see a larger dynamic aperture reduction for working point (28.685, $29.695)$ than that for working point $(28.695,29.685)$. For the nominal working point $(28.685,29.695)$, full head-on beam-beam compensation gives $0.4 \sigma$ more reductions in the minimum and angle averaged dynamic apertures than half head-on beam-beam compensation

In conclusion, reductions in $10^{6}$ turn dynamic apertures are seen with head-on beam-beam compensation. For the nominal working point $(28.685,29.695)$, full head-on beam-beam compensation gives a $0.4 \sigma$ larger reductions in the minimum and angle averaged dynamic apertures than half head-on beam-beam compensation. This is in agreement with the above qualitative analysis using short-term tune diffusion and the Lyapunov exponent.

\section{Dynamic apertures in tune scan}

In this section we continue to calculate $10^{6}$ turn dynamic apertures with beam-beam compensation in a tune scan. The tune scan is carried out along the diagonal in the tune space and the step size of tune change is 0.005 .

With half head-on beam-beam compensation, we calculate dynamic apertures for working points $(28.685$, $29.695)$ and $(28.680,29.690)$ above the diagonal and for working points $(28.695,29.685)$ and $(28.690,29.680)$ below the diagonal. With full head-on beam-beam compensation, we calculate dynamic apertures for working points $(28.685,29.695),(28.680,29.690)$ and $(28.675,29.685)$ above diagonal and for working points $(28.695$, $28.685),(28.690,28.680)$ and $(28.685,28.675)$ below diagonal.

Figure 11 and Fig. 12 show the dynamic apertures in the tune scan with half and full head-on beam-beam compensations respectively. Table 3 list all the calculated dynamic apertures in the tune scan. For each working point dynamic apertures are calculated for on- and off-momentum particles. The relative momentum deviation for off-momentum particles is $\Delta p / p_{0}=0.0005$.

From Fig. 11 and Tab. 3, for cases with half the head-on beam-beam compensation, there are very small changes in the phase averaged dynamic apertures in the tune scan. These changes are typically less than $0.2 \sigma$, which is close to the resolution of our dynamic aperture searching. The difference in the minimum 
Table 2: Dynamic apertures in $10^{6}$ turn trackings

\begin{tabular}{|c|c|c|c|c|c|c|c|c|c|c|c|}
\hline 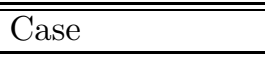 & $\overline{\mathrm{D}\left(5^{\circ}\right)}$ & $\overline{\mathrm{D}\left(15^{\circ}\right)}$ & $\overline{\mathrm{D}\left(25^{\circ}\right)}$ & $\overline{\mathrm{D}\left(35^{\circ}\right)}$ & 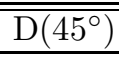 & $\overline{\mathrm{D}\left(55^{\circ}\right)}$ & $\overline{\mathrm{D}\left(65^{\circ}\right)}$ & $\overline{\mathrm{D}\left(75^{\circ}\right)}$ & $\overline{\mathrm{D}\left(85^{\circ}\right)}$ & $\overline{\overline{D_{\min }}}$ & $\begin{array}{l}D_{a v g} \\
\end{array}$ \\
\hline \multicolumn{12}{|l|}{ WP685695-dp0: } \\
\hline NoBB & 9.3 & 9.3 & 8.0 & 8.2 & 7.9 & 7.3 & 8.4 & 9.0 & 9.2 & 7.3 & 8.3 \\
\hline BB & 6.5 & 6.4 & 5.9 & 6.1 & 6.5 & 6.5 & 5.7 & 7.1 & 7.1 & 5.7 & 6.4 \\
\hline $\mathrm{HBBC}$ & 6.3 & 7.9 & 6.0 & 5.6 & 6.2 & 6.0 & 5.8 & 6.0 & 6.8 & 5.6 & 6.2 \\
\hline FBBC & 6.3 & 6.3 & 6.1 & 5.7 & 5.5 & 5.1 & 4.6 & 6.2 & 6.0 & 4.6 & 5.7 \\
\hline \multicolumn{12}{|l|}{ WP685695-dp5: } \\
\hline NoBB & 8.0 & 8.2 & 8.4 & 8.7 & 8.2 & 7.6 & 8.0 & 9.9 & 10.3 & 7.6 & 8.5 \\
\hline BB & 6.6 & 6.4 & 6.5 & 6.8 & 6.9 & 6.4 & 6.5 & 8.0 & 7.7 & 6.4 & 6.8 \\
\hline $\mathrm{HBBC}$ & 6.6 & 6.6 & 6.5 & 6.5 & 6.0 & 6.5 & 5.7 & 6.8 & 8.2 & 5.7 & 6.5 \\
\hline FBBC & 6.4 & 6.6 & 6.4 & 6.1 & 5.3 & 5.7 & 5.8 & 6.8 & 6.7 & 5.3 & 6.1 \\
\hline \multicolumn{12}{|l|}{ WP695685-dp0: } \\
\hline NoBB & 10.1 & 8.8 & 8.0 & 7.3 & 8.0 & 8.4 & 8.2 & 7.8 & 9.0 & 7.3 & 8.2 \\
\hline BB & 9.3 & 7.1 & 6.9 & 5.6 & 5.4 & 5.7 & 7.3 & 6.5 & 6.1 & 5.4 & 6.5 \\
\hline HBBC & 9.2 & 7.2 & 6.5 & 5.6 & 5.7 & 5.7 & 7.1 & 6.5 & 6.1 & 5.6 & 6.5 \\
\hline FBBC & 9.0 & 6.6 & 5.4 & 5.3 & 5.5 & 5.7 & 5.7 & 6.3 & 5.7 & 5.3 & 5.9 \\
\hline \multicolumn{12}{|l|}{ WP695685-dp5: } \\
\hline NoBB & 10.5 & 8.6 & 8.8 & 8.0 & 8.8 & 9.2 & 8.8 & 8.6 & 8.4 & 8.0 & 8.8 \\
\hline BB & 9.8 & 7.7 & 6.9 & 6.1 & 6.1 & 6.2 & 7.2 & 7.2 & 6.8 & 6.1 & 6.9 \\
\hline HBBC & 9.5 & 7.3 & 6.6 & 5.8 & 6.5 & 6.2 & 6.9 & 7.1 & 6.9 & 5.8 & 6.8 \\
\hline FBBC & 10.1 & 7.1 & 6.0 & 6.0 & 6.1 & 5.8 & 7.1 & 6.7 & 6.9 & 5.8 & 6.6 \\
\hline
\end{tabular}

dynamic apertures are also small in the tune scan. In most cases, the minimum dynamic apertures differ by $0.1 \sigma$, expect for the off-momentum particles above diagonal which show a $0.3 \sigma$ change.

With full head-on beam-beam compensation, the changes in the tune scan in the phase averaged dynamic apertures are around $0.1 \sigma$. There is only one case with a larger phase averaged dynamic aperture, for onmomentum particles with nominal working point $(28.690,29.680)$.

To conclude this section, there are no discernible differences in the $10^{6}$ turn dynamic apertures in the above tune scan. In our tune scan, the lower tunes with compensation are all above 0.67 to avoid the third order resonances at $2 / 3$.

\section{Possibility of bunch intensity $N_{p}=3.0 \times 10^{11}$}

In this section we evaluate the possibility of increasing the bunch intensity to $N_{p}=3.0 \times 10^{11}$ which gives about -0.03 total beam-beam tune shift from interactions at IP6 and IP8. We compensate two-thirds of the total beam-beam tune shift with an e-lens which gives the final tune shift for the bunch core of about -0.01 . With the $2 / 3$ head-on beam-beam compensation for $N_{p}=3.0 \times 10^{11}$, the required electron beam intensity of the e-lens is $N_{e}=4.0 \times 10^{11}$. If there is no significant change in the proton beam lifetime or emittance growth with $N_{p}=3.0 \times 10^{11}$ and $2 / 3$ head-on beam-beam compensation, the luminosity will be doubled.

Figure 13 and Fig. 14 show the tune footprint, tune diffusion, Lyapunov exponent and dynamic aperture for working points $(28.685,29.695)$ and $(28.695,29.685)$ with bunch intensity $N_{p}=3.0 \times 10^{11}$ and $2 / 3$ head-on beam-beam compensation respectively. Table 4 lists the calculated $10^{6}$ turn dynamic apertures.

From Fig. 13 and Fig. 14, with bunch intensity $N_{p}=3.0 \times 10^{11}$ and $2 / 3$ head-on beam-beam compensation, the tune shifts for the bunch core are about 0.01 . Tune footprint foldings happen in both radial and azimuthal directions from very small particle amplitudes. From tune diffusion and Lyapunov exponent calculation, black dots are seen starting from $2.5 \sigma$.

From Tab. 4, with $N_{p}=3.0 \times 10^{11}$ and $2 / 3$ head-on beam-beam compensation, for the nominal working point $(28.695,29.685)$, both the minimum and phase averaged dynamic apertures for off-momentum particles dropped by $0.4 \sigma$ compared to the case of $N_{p}=2.0 \times 10^{11}$ and with full head-on beam-beam compensation. For the nominal working point $(28.685,29.695)$ the difference between these two cases is less than $0.2 \sigma$.

To conclude, with $N_{p}=3.0 \times 10^{11}$ and $2 / 3$ head-on beam-beam compensation, there is a $0.4 \sigma$ drop in the $10^{6}$ turn dynamic aperture for the nominal working point $(28.695,29.685)$, compared to the case with $N_{p}=2.0 \times 10^{11}$ and full head-on beam-beam compensation. For the nominal working point $(28.685,29.695)$, 
Table 3: $10^{6}$ turn dynamic apertures in the tune scan

\begin{tabular}{|c|c|c|c|c|c|c|c|c|c|c|c|}
\hline Case & $\overline{\mathrm{D}\left(5^{\circ}\right)}$ & $\bar{D}\left(15^{\circ}\right)$ & $\overline{\mathrm{D}\left(25^{\circ}\right)}$ & $\mathrm{D}\left(35^{\circ}\right)$ & $\overline{\mathrm{D}\left(45^{\circ}\right)}$ & $\overline{\mathrm{D}\left(55^{\circ}\right)}$ & $\overline{\mathrm{D}\left(65^{\circ}\right)}$ & $\overline{\mathrm{D}\left(75^{\circ}\right)}$ & $\mathrm{D}\left(85^{\circ}\right)$ & $\overline{D_{\min }}$ & $\overline{\bar{D} D_{a v g}}$ \\
\hline \multicolumn{12}{|l|}{ Half BBC: } \\
\hline WP685695-dp0 & 6.3 & 7.9 & 6.0 & 5.6 & 6.2 & 6.0 & 5.8 & 6.0 & 6.8 & 5.6 & 6.2 \\
\hline WP680690-dp0 & 6.6 & 6.5 & 6.5 & 6.6 & 5.7 & 5.5 & 6.2 & 6.9 & 7.2 & 5.5 & 6.3 \\
\hline WP680690-dp5 & 6.9 & 6.8 & 6.5 & 6.3 & 6.1 & 6.0 & 5.3 & 6.9 & 7.9 & 5.3 & 6.4 \\
\hline WP695685-dp0 & 9.2 & 7.2 & 6.5 & 5.6 & 5.7 & 5.7 & 7.1 & 6.5 & 6.1 & 5.6 & 6.5 \\
\hline WP685680-dp5 & 10.2 & 6.6 & 5.7 & 6.3 & 6.2 & 6.9 & 7.9 & 7.2 & 6.9 & 5.7 & 7.0 \\
\hline \multicolumn{12}{|l|}{ Full BBC: } \\
\hline WP685695-dp0 & 6.3 & 6.3 & 6.1 & 5.7 & 5.5 & 5.1 & 4.6 & 6.2 & 6.0 & 4.6 & 5.7 \\
\hline WP680690-dp0 & 6.5 & 6.4 & 6.0 & 5.8 & 5.2 & 5.6 & 5.3 & 6.0 & 6.6 & 5.2 & 5.8 \\
\hline WP675685-dp0 & 6.5 & 6.4 & 6.3 & 5.8 & 5.2 & 5.5 & 5.2 & 5.9 & 6.2 & 5.2 & 5.8 \\
\hline WP695685-dp0 & 9.0 & 6.6 & 5.4 & 5.3 & 5.5 & 5.7 & 5.7 & 6.3 & 5.7 & 5.3 & 5.9 \\
\hline WP690680-dp0 & 8.2 & 6.6 & 5.5 & 5.6 & 5.8 & 6.1 & 7.1 & 6.5 & 6.0 & 5.5 & 6.3 \\
\hline WP685675-dp0 & 8.0 & 6.3 & 4.9 & 5.3 & 5.4 & 6.3 & 6.5 & 6.2 & 5.8 & 4.9 & 6.0 \\
\hline WP695685-dp5 & 10.1 & 7.1 & 6.0 & 6.0 & 6.1 & 5.8 & 7.1 & 6.7 & 6.9 & 5.8 & 6.6 \\
\hline WP690680-dp5 & 7.6 & 6.2 & 6.4 & 5.2 & 5.7 & 6.2 & 7.8 & 7.2 & 6.9 & 5.2 & 6.5 \\
\hline WP685675-dp5 & 7.6 & 6.0 & 5.7 & 5.9 & 6.2 & 6.8 & 7.3 & 6.9 & 6.7 & 5.7 & 6.5 \\
\hline
\end{tabular}

Table 4: $10^{6}$ turn dynamic apertures with bunch intensity $3.0 \times 10^{11}$

\begin{tabular}{lccccccccccc}
\hline \hline Case & $\mathrm{D}\left(5^{\circ}\right)$ & $\mathrm{D}\left(15^{\circ}\right)$ & $\mathrm{D}\left(25^{\circ}\right)$ & $\mathrm{D}\left(35^{\circ}\right)$ & $\mathrm{D}\left(45^{\circ}\right)$ & $\mathrm{D}\left(55^{\circ}\right)$ & $\mathrm{D}\left(65^{\circ}\right)$ & $\mathrm{D}\left(75^{\circ}\right)$ & $\mathrm{D}\left(85^{\circ}\right)$ & $D_{\min }$ & $D_{a v g}$ \\
\hline WP685695-dp0 & 6.1 & 6.1 & 5.6 & 5.4 & 5.4 & 5.4 & 5.7 & 5.7 & 5.6 & 5.4 & 5.6 \\
WP685695-dp5 & 6.5 & 6.4 & 6.0 & 5.4 & 6.1 & 5.8 & 5.7 & 5.5 & 6.8 & 5.4 & 5.9 \\
WP695685-dp0 & 9.3 & 6.3 & 5.7 & 5.1 & 5.5 & 5.4 & 6.1 & 6.2 & 5.8 & 5.1 & 6.0 \\
WP695685-dp5 & 7.1 & 7.2 & 5.8 & 5.2 & 6.1 & 6.0 & 6.3 & 6.6 & 6.5 & 5.2 & 6.2 \\
\hline \hline
\end{tabular}

the difference between these two cases is less than $0.2 \sigma$.

\section{Discussion of footprint folding}

In this section we discuss the cause of tune footprint foldings. As an example we calculate tune shifts versus horizontal amplitude for on-momentum particles at the nominal working point $(28.685,29.695)$. All the detunings shown below are given with respect to the tunes for particles with zero amplitude.

The top-left plot in Fig. 15 shows the horizontal detuning only from the nonlinear magnetic fields in the lattice. The top-right plot in Fig. 15 shows the detuning only from p-p beam-beam interactions at IP6 and IP8. The proton bunch intensity is $N_{p}=3.0 \times 10^{11}$. There is no clear tune folding in the horizontal plane in all phase angles. The detuning increases or decreases with the particle amplitude monotonically. This means that nonlinearities from the lattice only or beam-beam interaction only will not cause tune footprint foldings.

When the nonlinear magnetic fields from lattice and the beam-beam interaction are both turned on, the total detunings are likely to fold at some amplitude depending on the strengths of both detunings. If the detuning from nonlinear magnetic fields is large and the the beam-beam detuning small, the tune footprint folding will occur at small amplitudes. If the detuning from nonlinear magnetic fields is small and the beam-beam detuning is large, the tune footprint folding will occur at larger amplitudes.

With both head-on beam-beam interactions and beam-beam compensation tune footprint foldings can occur even without lattice nonlinearities. The phase advances between the beam-beam interaction and compensation locations clearly play an important role. Proper phase advances between beam-beam interaction 
and compensation will push the tune footprint folding to a higher amplitude and therefore will yield a larger dynamic aperture.

Tune footprint foldings can occur in the azimuthal as well as in the radial directions in the $\left(x / \sigma_{x}, y / \sigma_{y}\right)$ plane. As an example, the bottom-left and bottom-right plots in Fig. 15 show the detunings with $N_{p}=$ $3.0 \times 10^{11}$ and $2 / 3$ head-on beam-beam compensation in the radial and azimuthal directions respectively. The foldings in the azimuthal direction is mainly caused by the different detunings from nonlinear magnetic fields in different phase angles. In our case, since the horizontal and vertical betatron amplitudes are almost same at the beam-beam interaction points, the beam-beam interactions and compensations will not cause the tune footprint foldings in the azimuthal direction.

To conclude, the tune footprint folding is caused by two or more mechanisms which provide detuning with different signs. These can be lattice nonlinearities and the beam-beam interaction, or the beam-beam interaction and beam-beam compensation. With head-on beam-beam compensation tune footprint foldings are likely to occur at a smaller amplitude.

\section{Tune diffusion map and Lyapunov exponent map}

In the above studies, both the tune diffusion and Lyapunov exponent analysis in short term tracking are used as indicators of long-term stability of single particle motion. In this section, we will compare these two tools.

The Lyapunov exponent has long been used to characterize regular and chaotic motion. The Lyapunov exponent analysis assumes that the distance between two particles originally very close in phase space will increase linearly or exponentially depending on whether the motion is regular or chaotic. It can be used in 4-D or 6-D tracking. The resolution of this method is limited by rounding errors. The tune diffusion analysis was introduced from celestial mechanics by Laskar in the late 1990's. This method depends on an accurate tune determination. It assumes that stable motion correlates with a small change in the betatron tunes over time. It is normally used in 4-D tracking since the longitudinal motion will disturb the betatron tune evaluation.

From Sec. 4 and Sec. 5, both tune diffusion and Lyapunov exponent map show resonance lines in the case of beam-beam interactions. The tune diffusion analysis is more powerful than the Lyapunov exponent to reveal resonance structures. Tune diffusion also shows resonance crossings or resonance overlapping in the case of full beam-beam compensations. The Lyapunov exponent method is sensitive to the diffusion in the spatial space.

However, sometimes tune diffusion and Lyapunov exponent maps tell a different story. For example, in the case of beam-beam compensation, and for particles below $4 \sigma$, the particles in the center of resonance stripes have larger tune diffusions than particle on the edges of resonance strips. While from Lyapunov exponent analysis, the particles in the center of resonance stripes have smaller Lyapunov exponents than those on the edge of the resonance strips. And in the Lyapunov exponent map, there is a larger red colored area around coordinates $(2,2)$ in the $\left(x / \sigma_{x}, y / \sigma_{y}\right)$ plane, while in the tune diffusion map these areas are colored blue. This example shows that sometimes resonances will not cause particle loss, especially at low amplitudes.

To conclude, tune diffusion is sensitive to the diffusion in the frequency domain while the Lyapunov exponent is sensitive in the diffusion in the spatial domain. The tune diffusion analysis is more powerful than Lyapunov exponent in revealing resonance structures. However, the Lyapunov exponent directly indicates which particle's motion is regular or chaotic. The Lyapunov exponent method can be used in medium-term tracking while tune diffusion is mostly limited to a short-term tracking. Both methods can be used to assess single particle long-term stability.

\section{Conclusion}

We calculated and compared the stability of single particle motion without and with head-on beam-beam compensation. From the calculated tune footprints, it is clear that head-on beam-beam can compensate effectively the beam-beam generated tune shift and tune spread.

Tune diffusion and Lyapunov exponents from 2048 turn tracking are used as indicators of long-term stability of single particle motion. Both studies show that head-on beam-beam compensation will stabilize the particles below $3 \sigma$. At the current working points head-on beam-beam compensation reduces the tune 
shifts and pulls the particles in the bunch core away from strong third order betatron resonances. The particles in the bunch core also see less nonlinear effects from the head-on beam-beam compensations.

Analysis of the tune diffusion and the Lyapunov exponent both show that head-on beam-beam compensation will negatively affect the stability of particles above $4 \sigma$. Head-on beam-beam compensation causes tune footprint foldings at small amplitudes, as well as resonance crossings and overlappings. Therefore, head-on beam-beam compensation reduces long-term dynamic aperture. Limited by the available CPU time and rounding errors, the dynamic aperture is calculated to $10^{6}$ turns only. In these studies, we observe that the dynamic apertures is slightly reduced with head-on beam-beam compensation.

\section{Acknowledgments}

We are thankful for the fruitful discussions with Y. Alexahin, J. Beebe-Wang, V. Shiltsev, C. Montag, G.

Robert-Demolaize, T. Roser, F. Schmidt, S. Tepikian and many others. This work is supported by the US DOE under contract No. DE-AC02-98CH10886.

\section{References}

[1] W. Fischer, Beam-beam and BTF, 2006 RHIC Accelerator Physics Experiments Workshop, November 2-3, 2005, BNL.

[2] V. Shiltsev, Electron lenses in Tevatron, RHIC and LHC, in the 2005 RHIC APEX workshop, BNL, November 2005.

[3] Y. Luo, W. Fischer, Outline of using an electron lens for the RHIC head-on beam-beam compensation, BNL C-AD AP Note 286, July 2007.

[4] V. Shiltsev, V. Danilov, D. Finley, and A. Sery, Phys. Rev. ST Accel. Beams 2, 071001 (1999).

[5] V. Shiltsev, Y. Alexahin, K. Bishofberger, V. Kamerdzhiev, G. Kuznetsov, and X.-L. Zhang, Phys. Rev. Lett. 99, 244801 (2007).

[6] Y. Luo, RHIC Head-On Beam-Beam Compensation with e-lens, in the LARP mini-workshop on beambea, compensation, SLAC, July 2007.

[7] Y. Luo, Progress in e-lens work for RHIC, in the LARP 9th collaboration meeting, SLAC, October 2007.

[8] SixTrack is availbale at http://frs.home.cern.ch/frs/.

[9] M. Bassetti and G.A. Erskine, Closed expression for the electricalfield of a two-dimensional Gaussian charge, CERN-ISR-TH/80-06.

[10] Sussix code is available at http://frs.home.cern.ch/frs/Source/other_tools/sussix/.

[11] J. Laskar, Frequency map analysis and particle accelerators, in the Proceedings of 1994 Particle Accelerator Conference, 2003.

[12] L.R. Evans and J. Gareyte, Beam-beam effects, CERN Yellow Report 87-03, 1987.

[13] A. Chao, Brief comments on nonlinear dynamics studies in storage rings, in the Proceedings of 1988 European Particle Accelerator Conference, 1988.

[14] J. Laskar, Physica 67 D, 257 (1993).

[15] J. Laskar and D. Robin, Part. Accel. 54, 183 (1996)

[16] M. Giovannozzi, W. Scandale and E. Todesco, Part. Accel. Vol 56, pp.195-225, 1997.

[17] E. Todesco and M. Giovannozzi, Dynamic aperture estimates and phase distortions in nonlinear betatronic motion, CERN SL(AP) 95-90, Phys. Rev. E 53, (1996), 4067.

[18] A. Chao and M. Tigner, Handbook of Accelerator Physics and Engineering, World Scientific, 1998. 


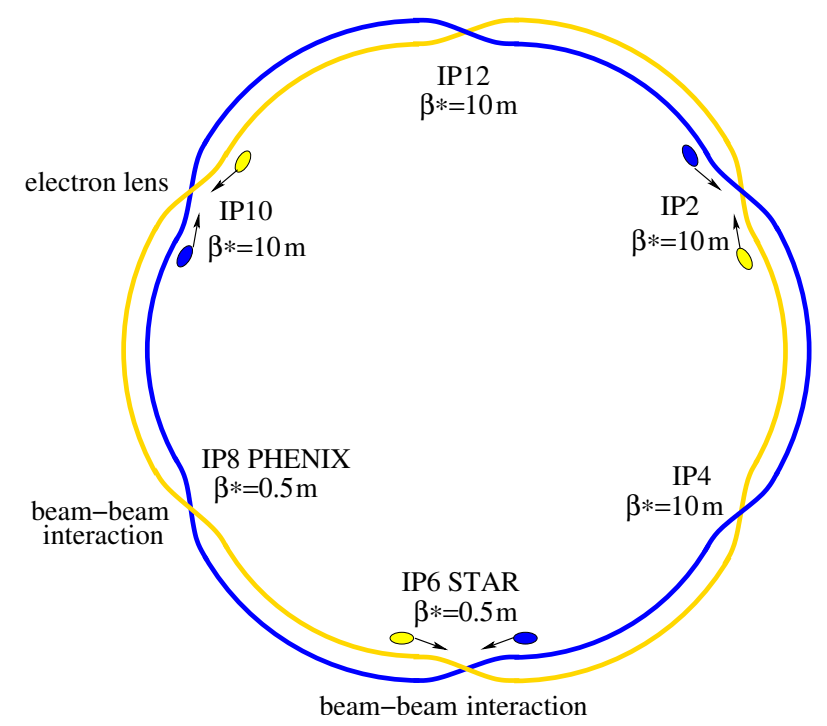

Figure 1: Layout of RHIC head-on beam-beam compensation.
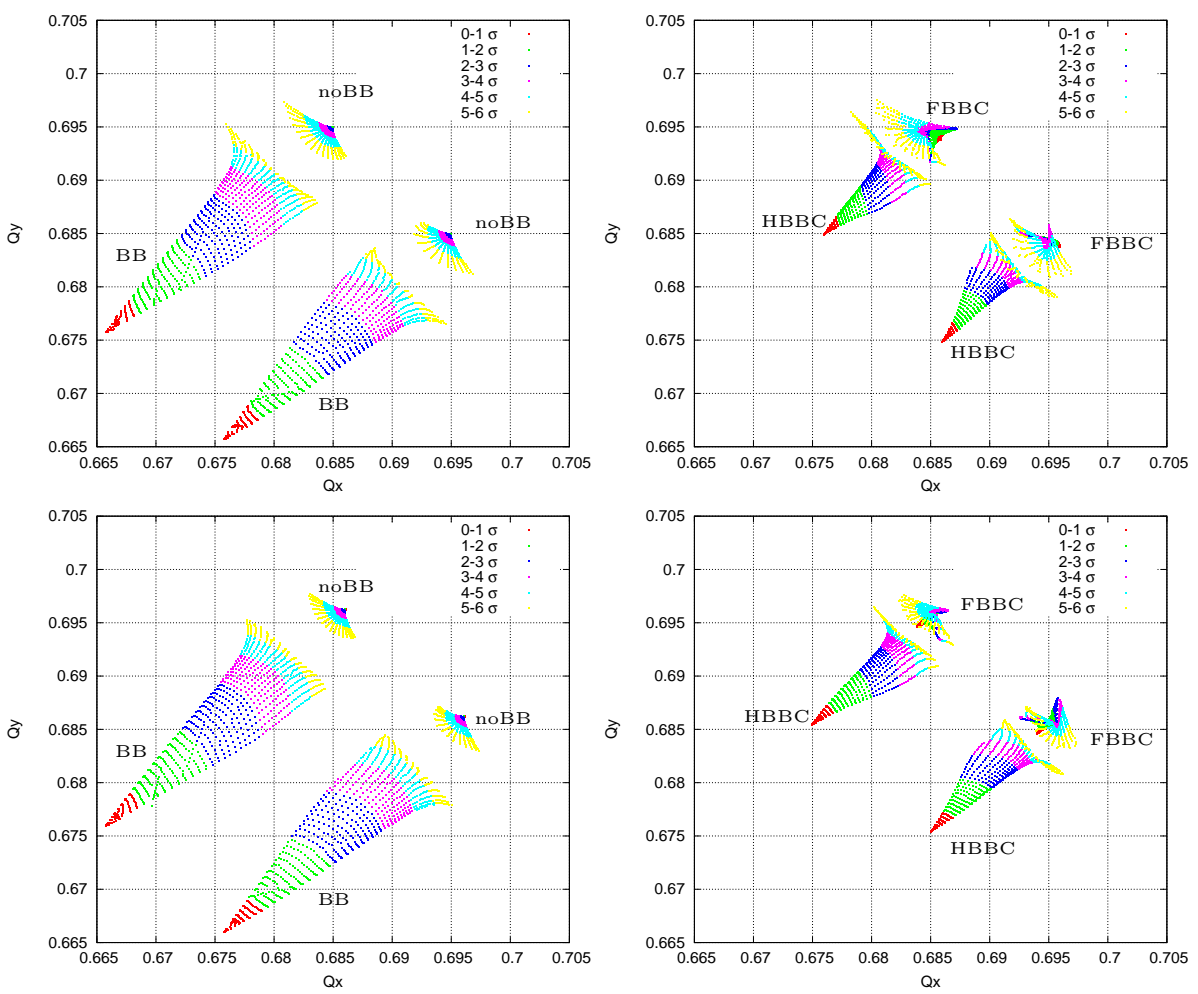

Figure 2: Tune footprints for both working points. Top-Left: on-momentum particles without and with BB; Top-right: on-momentum particles with half and full BB compensations. Bottom-left: off-momentum particles without and with BB; Bottom-right: off-momentum particles with half and full BB compensations. 

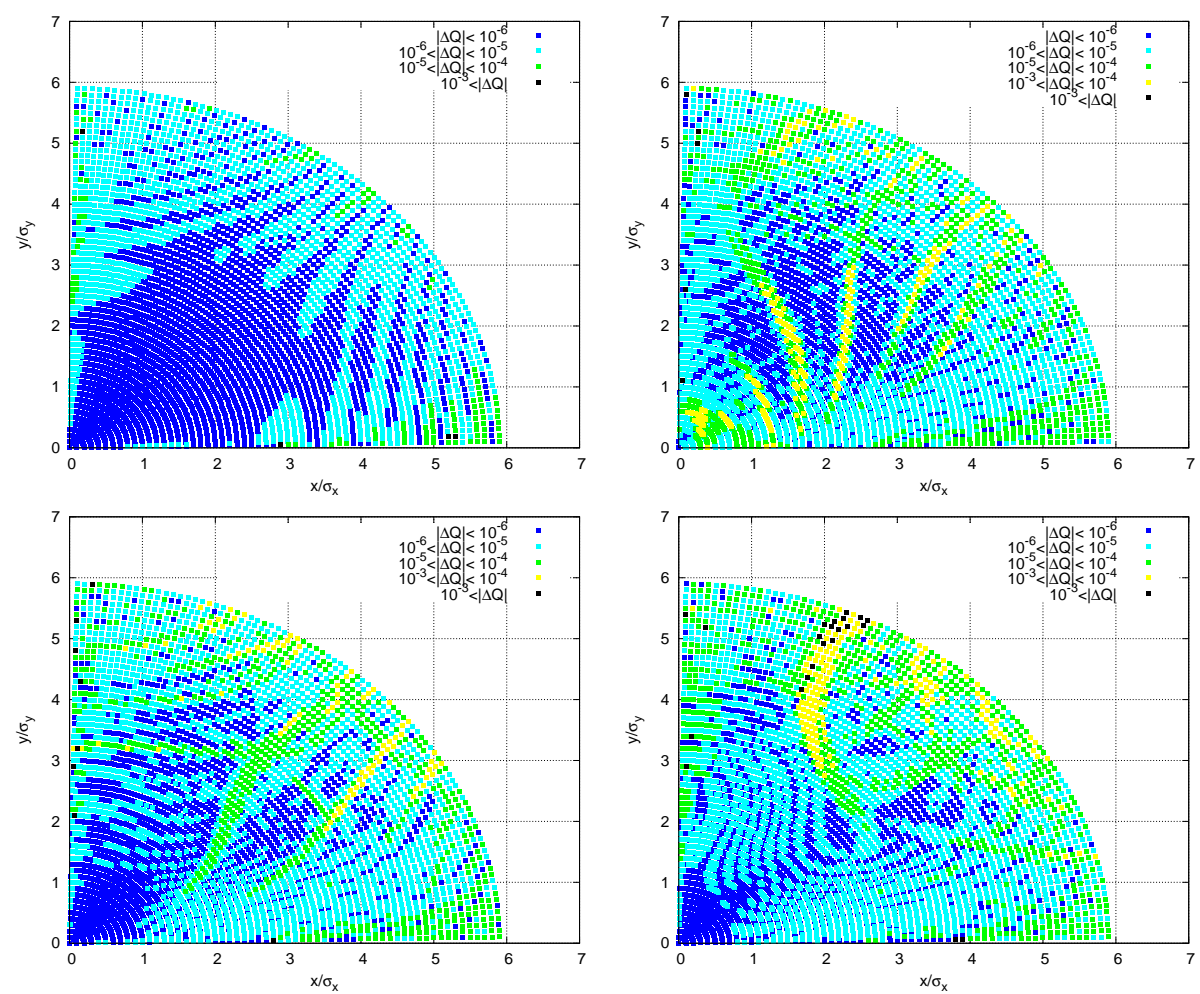

Figure 3: Tune diffusion maps of on-momentum particles for working point (28.685, 29.695): Top-left: without BB; Top-right: with BB; Bottom-left: with BB and half BB compensation; Bottom-right: with BB and full BB compensation.
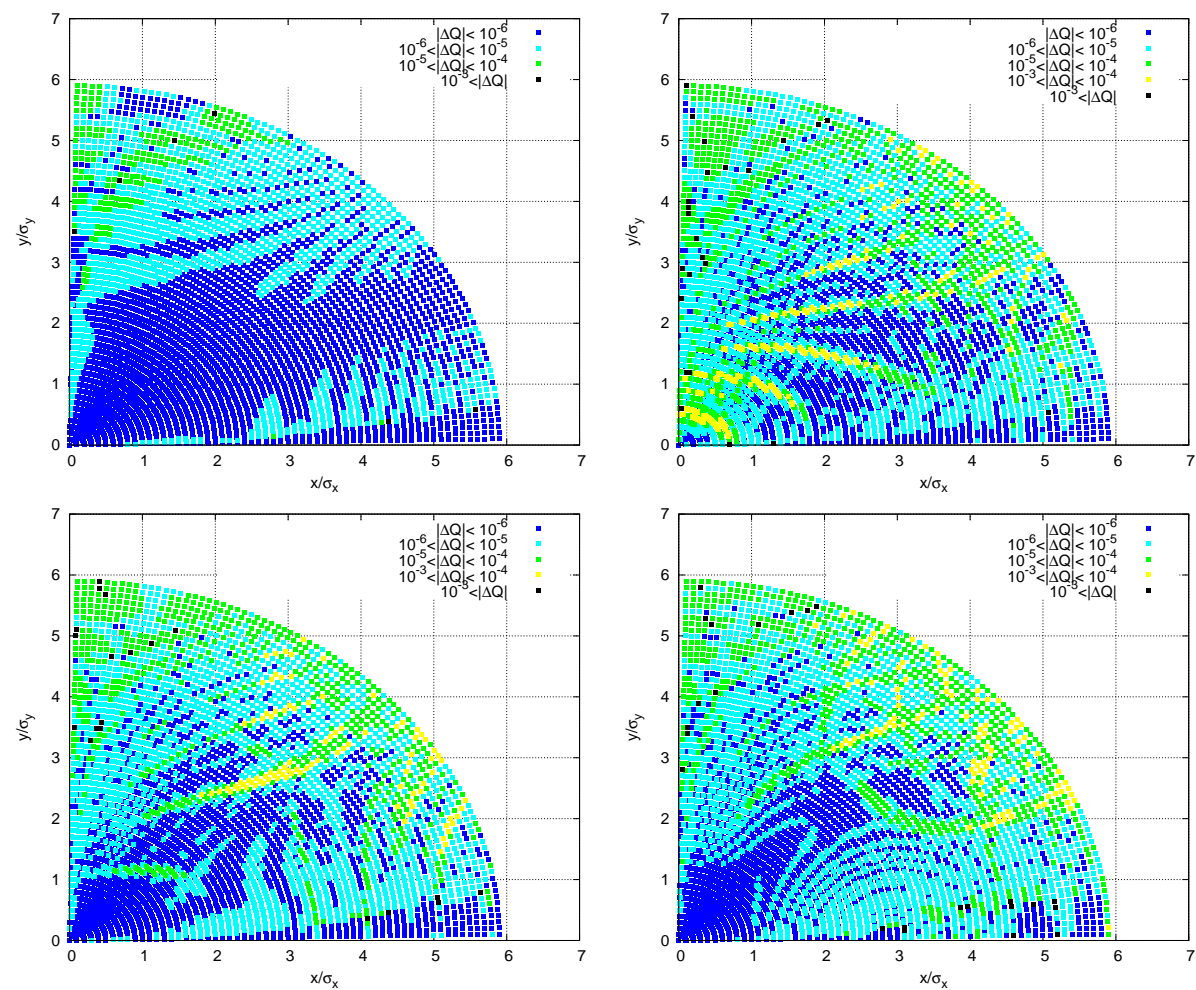

Figure 4: Tune diffusion maps of on-momentum particles for working point (28.695, 29.685): Top-left: without BB; Top-right: with BB; Bottom-left: with BB and half BB compensation; Bottom-right: with BB and full BB compensation. 

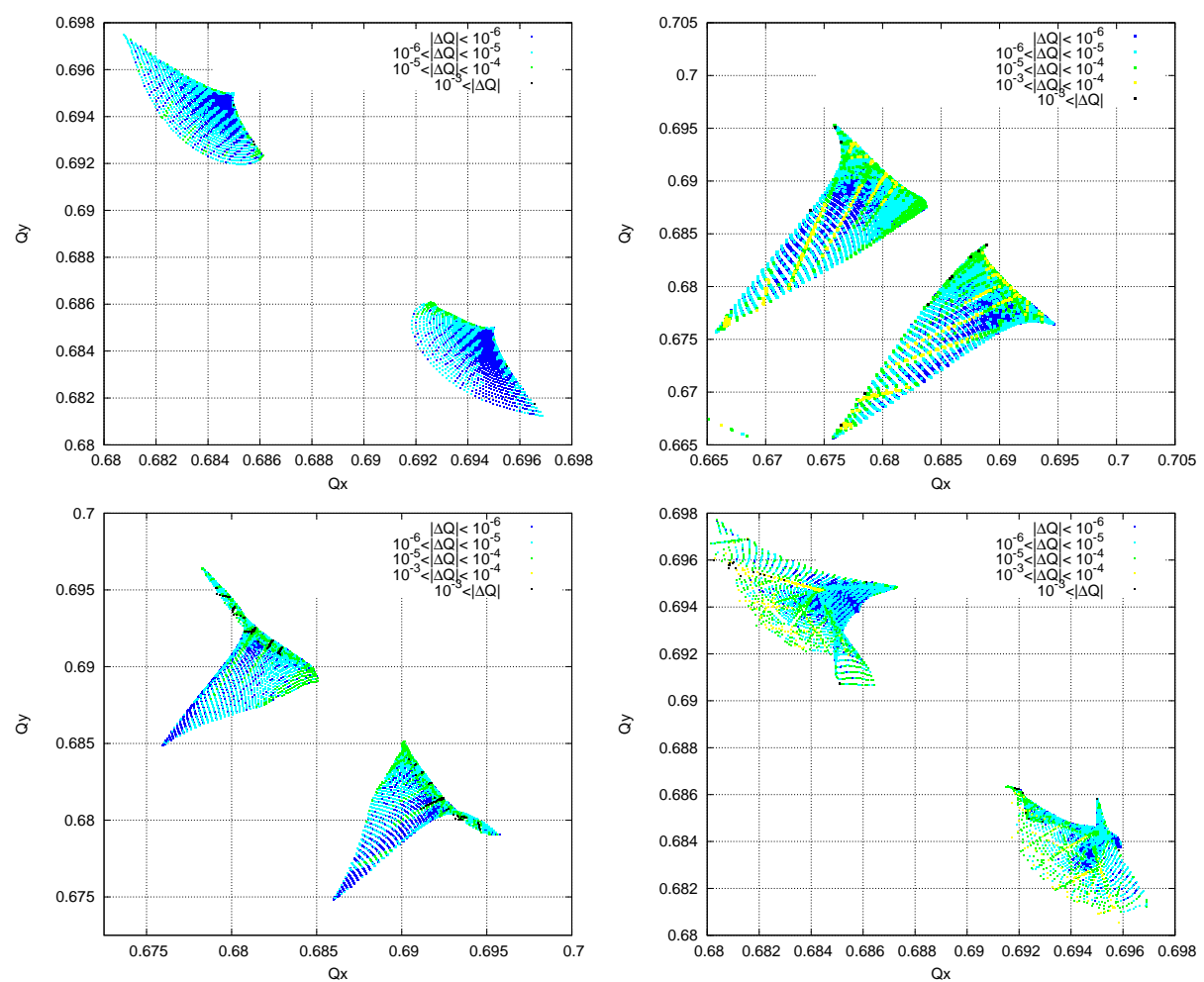

Figure 5: Tune diffusion maps of on-momentum particles in tune space for both working points: Top-left: without BB; Top-right: with BB; Bottom-left: with BB and half BB compensation; Bottom-right: with BB and full $\mathrm{BB}$ compensation.
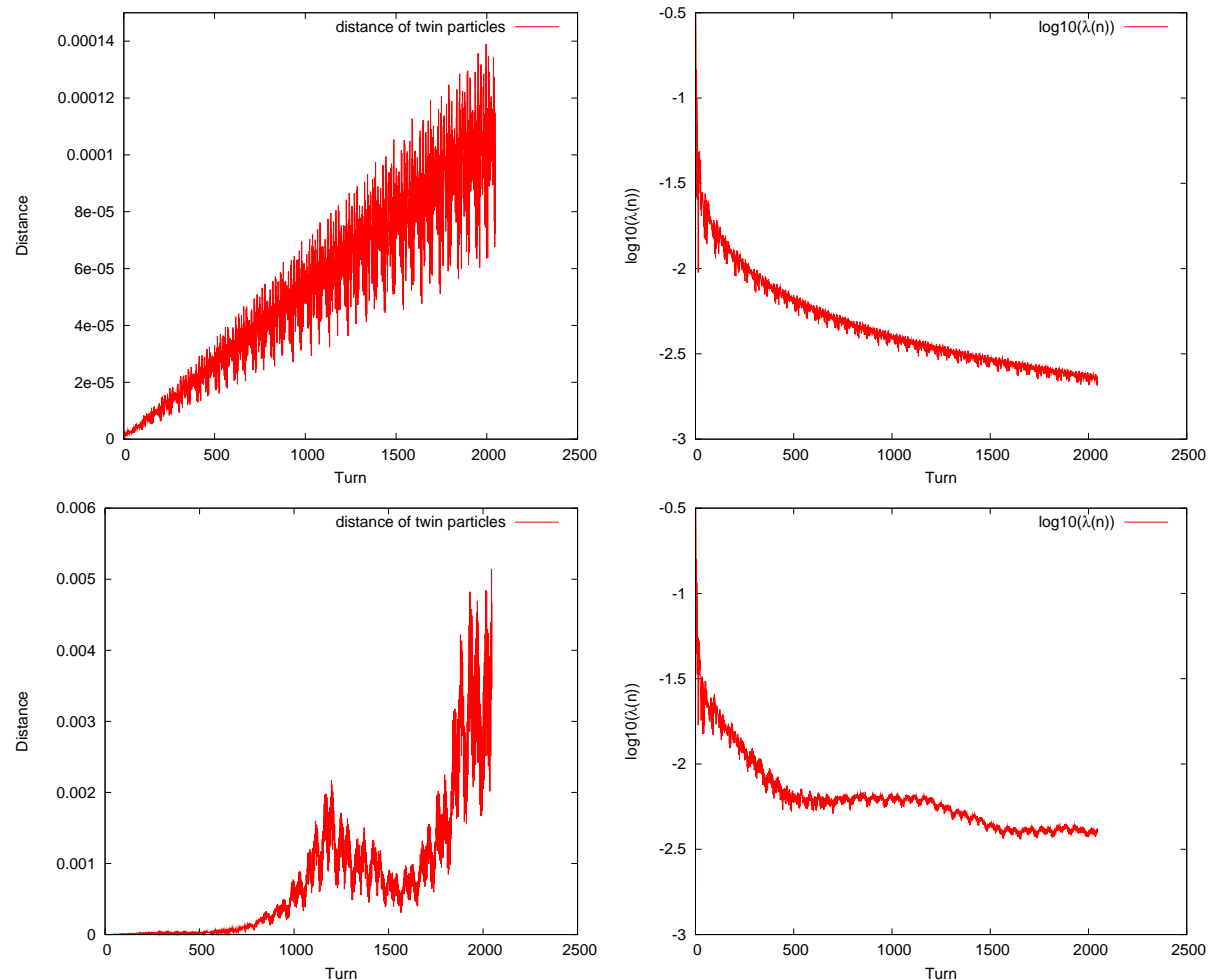

Figure 6: Example of Lyapunov exponents: Top-left and Top-right are the distance and $\lambda(n)$ of regular particle motion; Bottom-left and Bottom-right are the distance and $\lambda(n)$ of chaotic particle motion. Tracking condition for the regular particle motion: WP685695-noBB, $6 \sigma / 45^{\circ}$; Tracking condition for the chaotic particle motion: WP685695-BB-FBBC, $6 \sigma / 45^{\circ}$. 

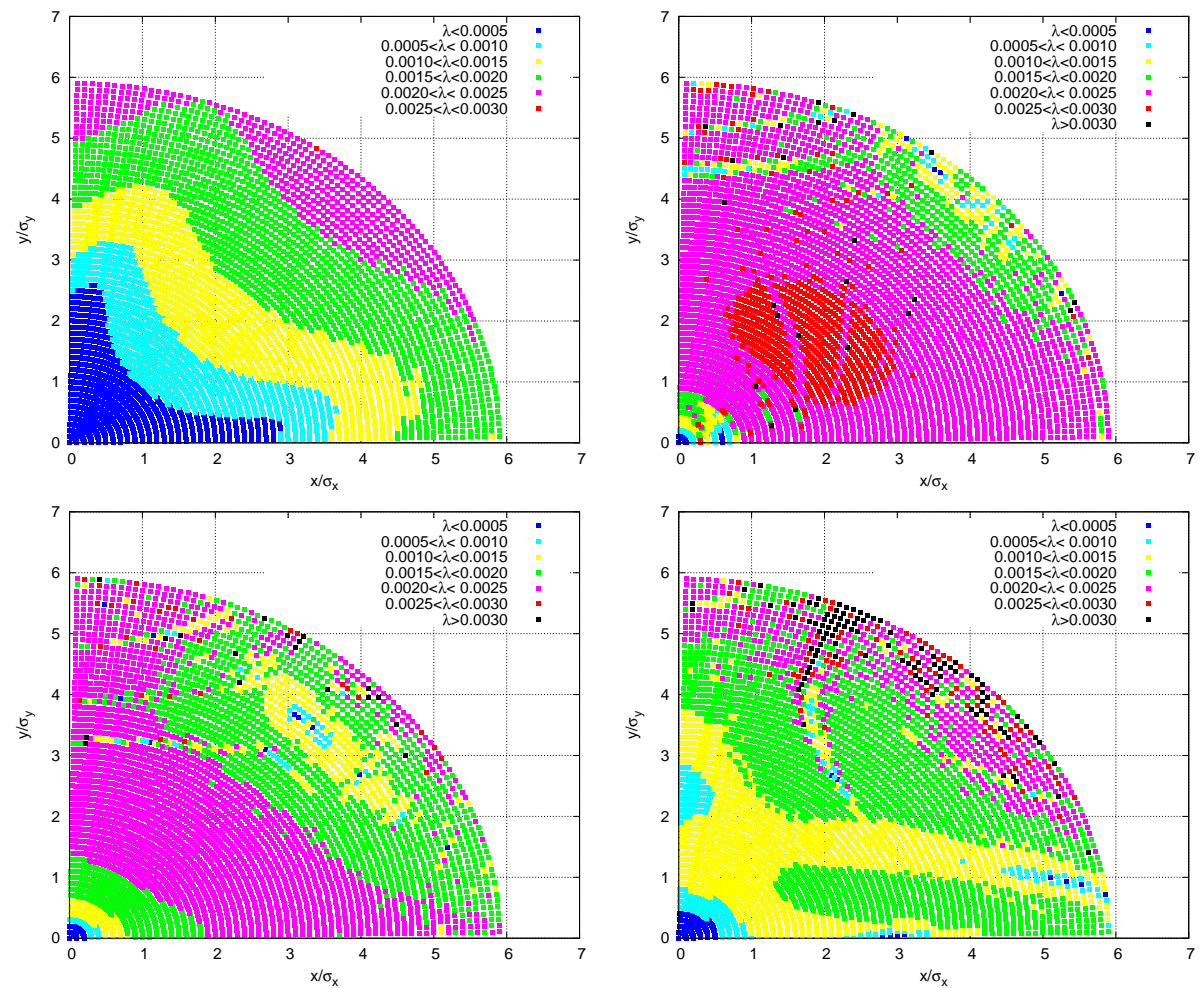

Figure 7: Lyapunov exponent maps of on-momentum particles for working point (28.685, 29.695): Top-left: without BB; Top-right: with BB; Bottom-left: with BB and half BB compensation; Bottom-right: with BB and full BB compensation.
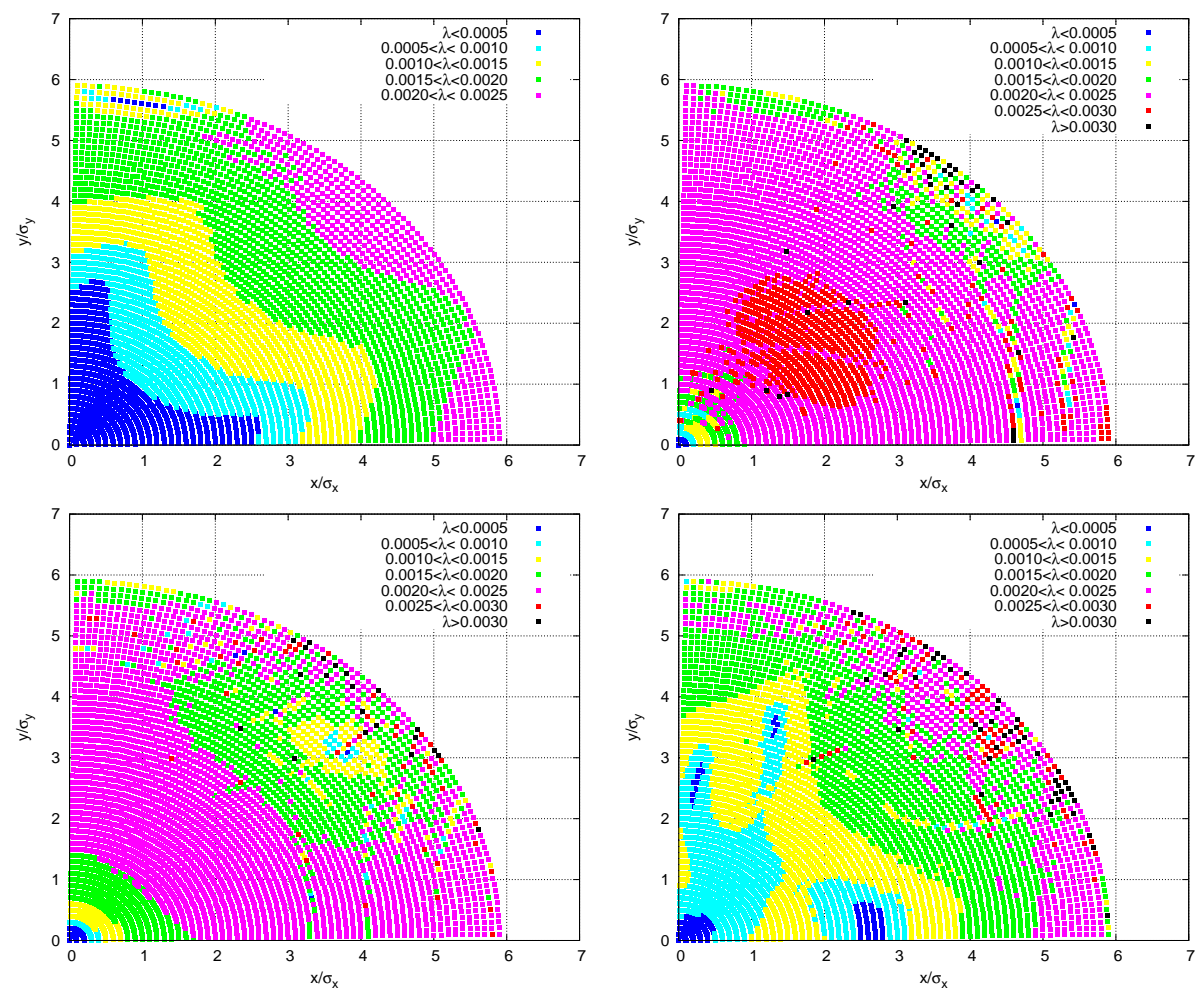

Figure 8: Lyapunov exponent maps of on-momentum particles for working point $(28.695,29.685)$ : Top-left: without BB; Top-right: with BB; Bottom-left: with BB and half BB compensation; Bottom-right: with BB and full BB compensation. 

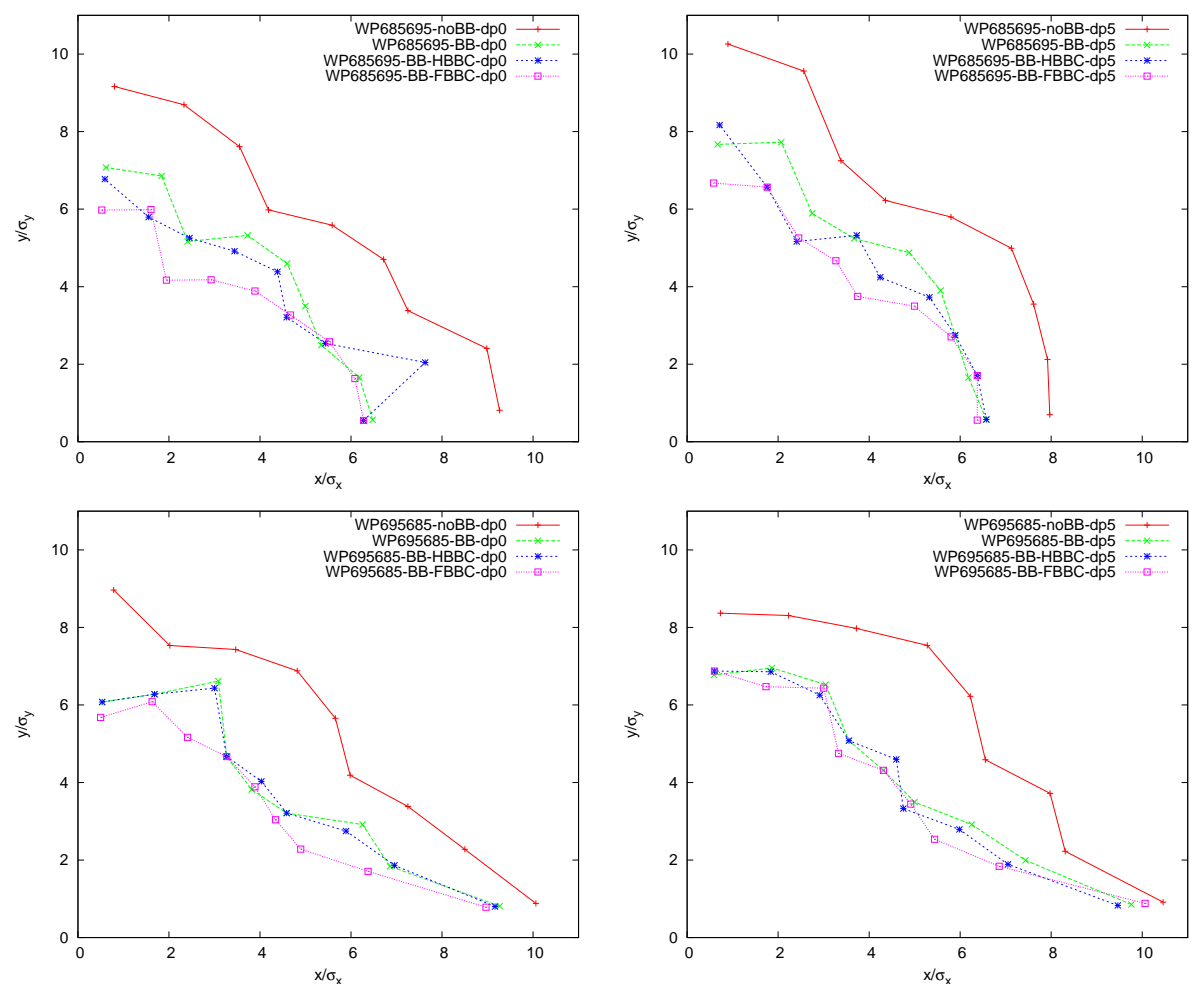

Figure 9: $10^{6}$ turn dynamic apertures. Top-left: on-momentum particles with working point $(28.685,29.695)$; Top-right: off-momentum particles with working point (28.685, 29.695); Bottom-left: on-momentum particles with working point $(28.695,29.685)$; Bottom-right: off-momentum particles with working point $(28.695$, 29.685).
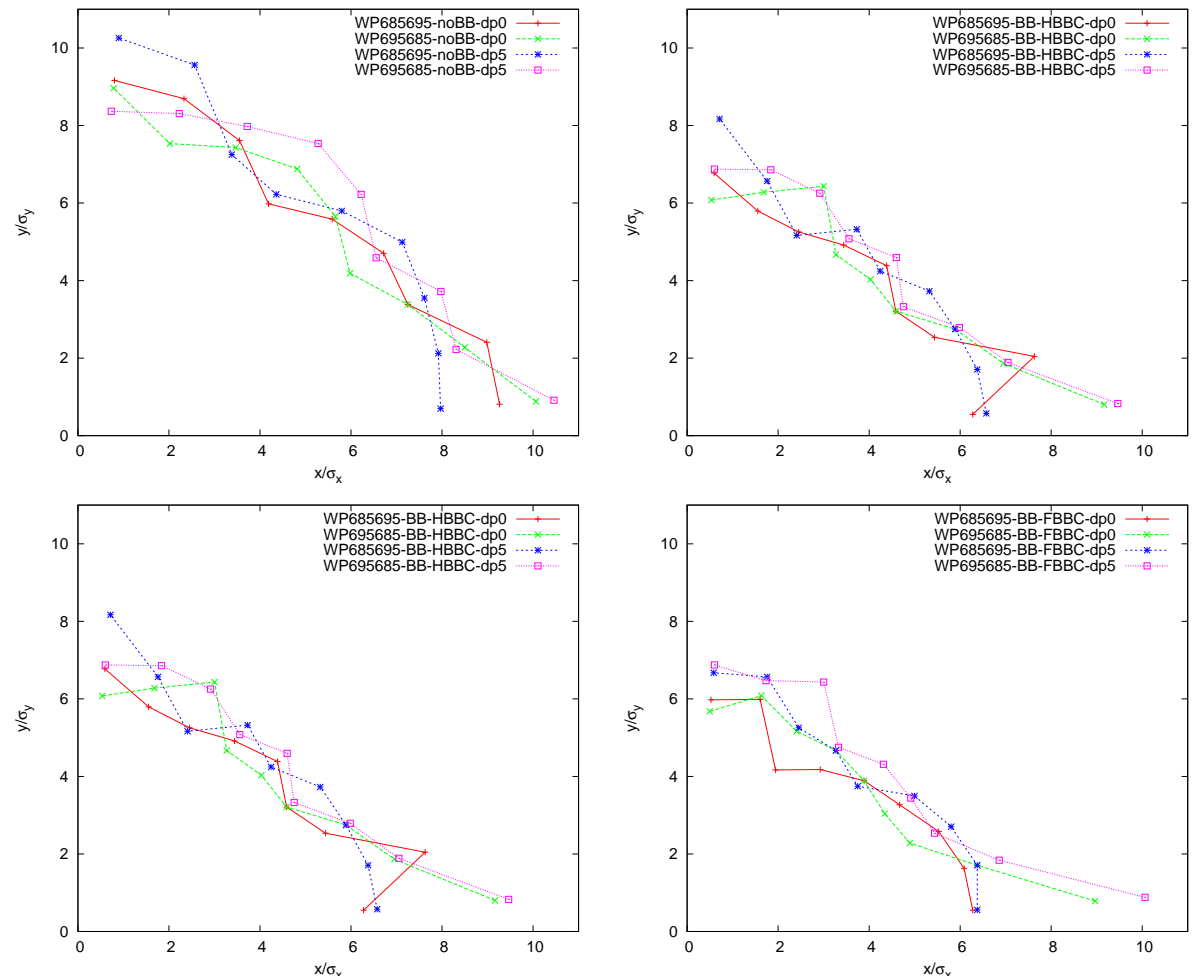

Figure 10: $10^{6}$ turn dynamic apertures. Top-left: without beam-beam; Top-right: with beam-beam interactions; Bottom-left: with beam-beam interactions and half beam-beam compensation; Bottom-right: with beam-beam interactions and full beam-beam compensation. 

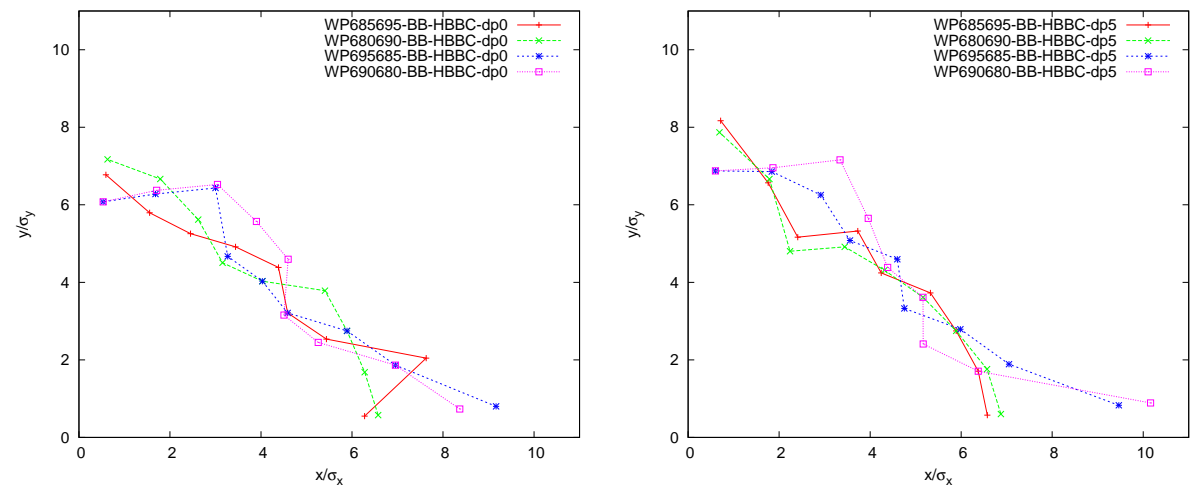

Figure 11: Tune scan with BB and half BBC.
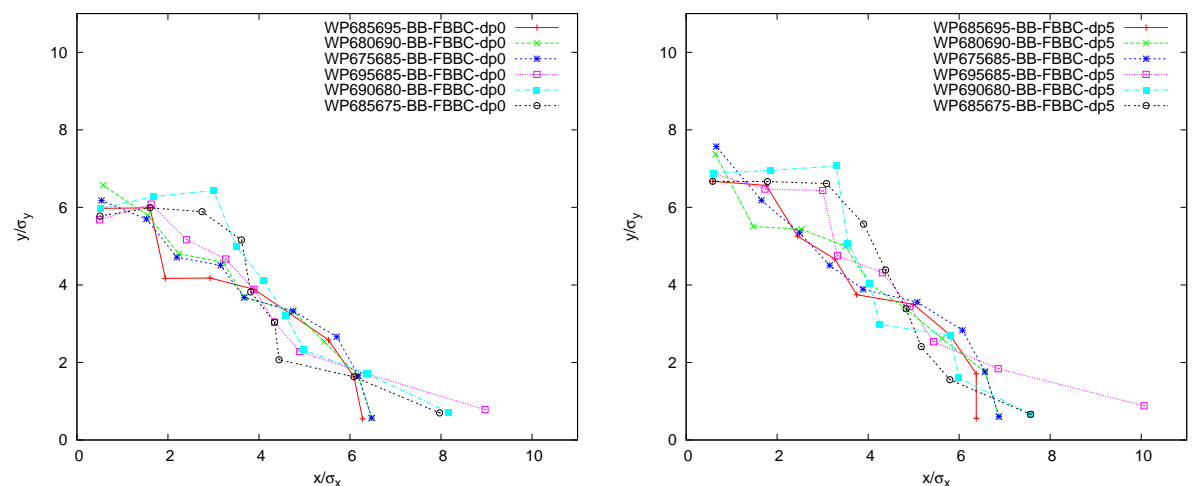

Figure 12: Tune scan with BB and full BBC.
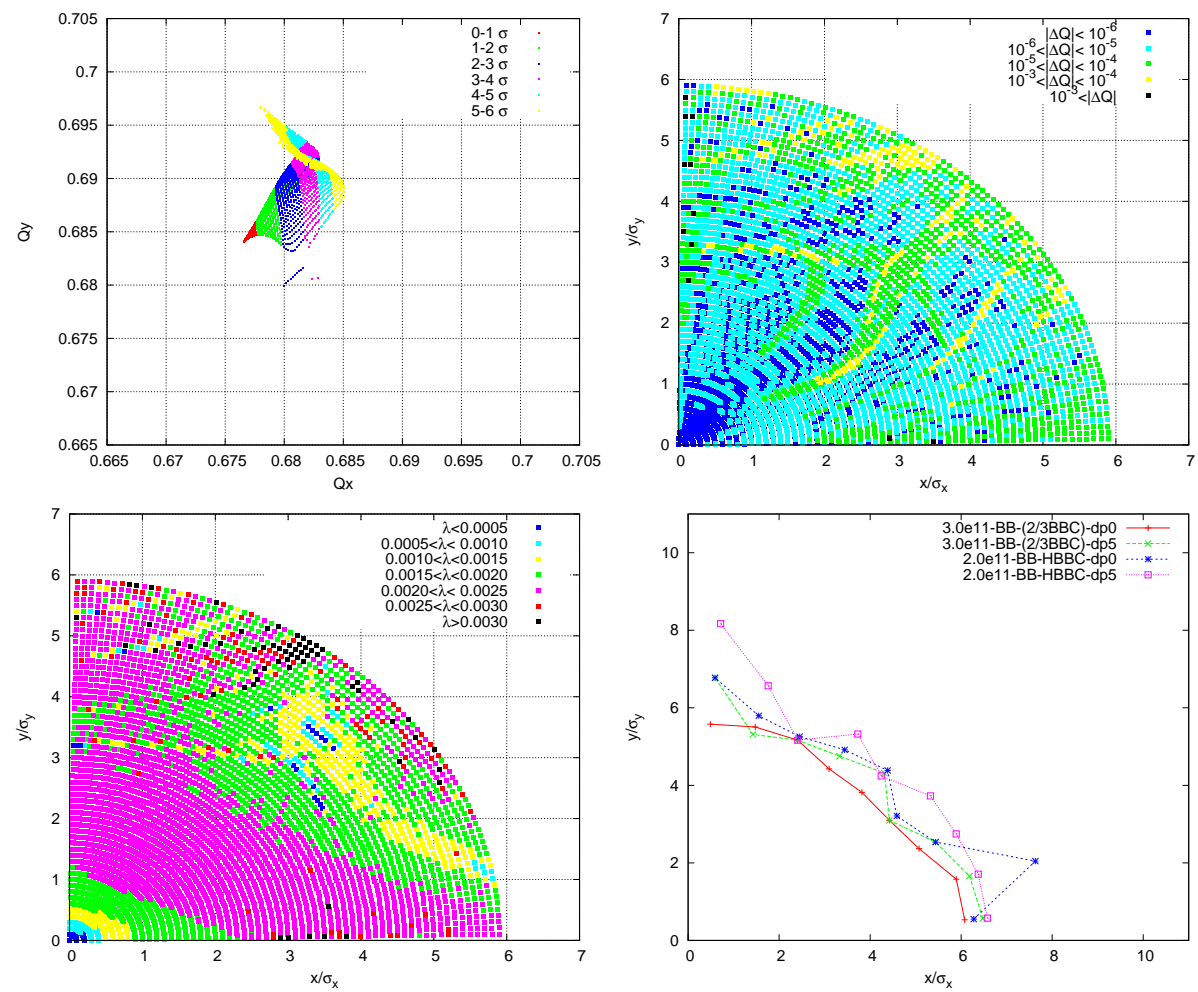

Figure 13: Tune footprint, tune diffusion, Lyapunov exponent, and dynamic apertures with $N_{p}=3.0 \times 10^{11}$ and $2 / 3$ BBC for working point $(28.685,29.695)$. 

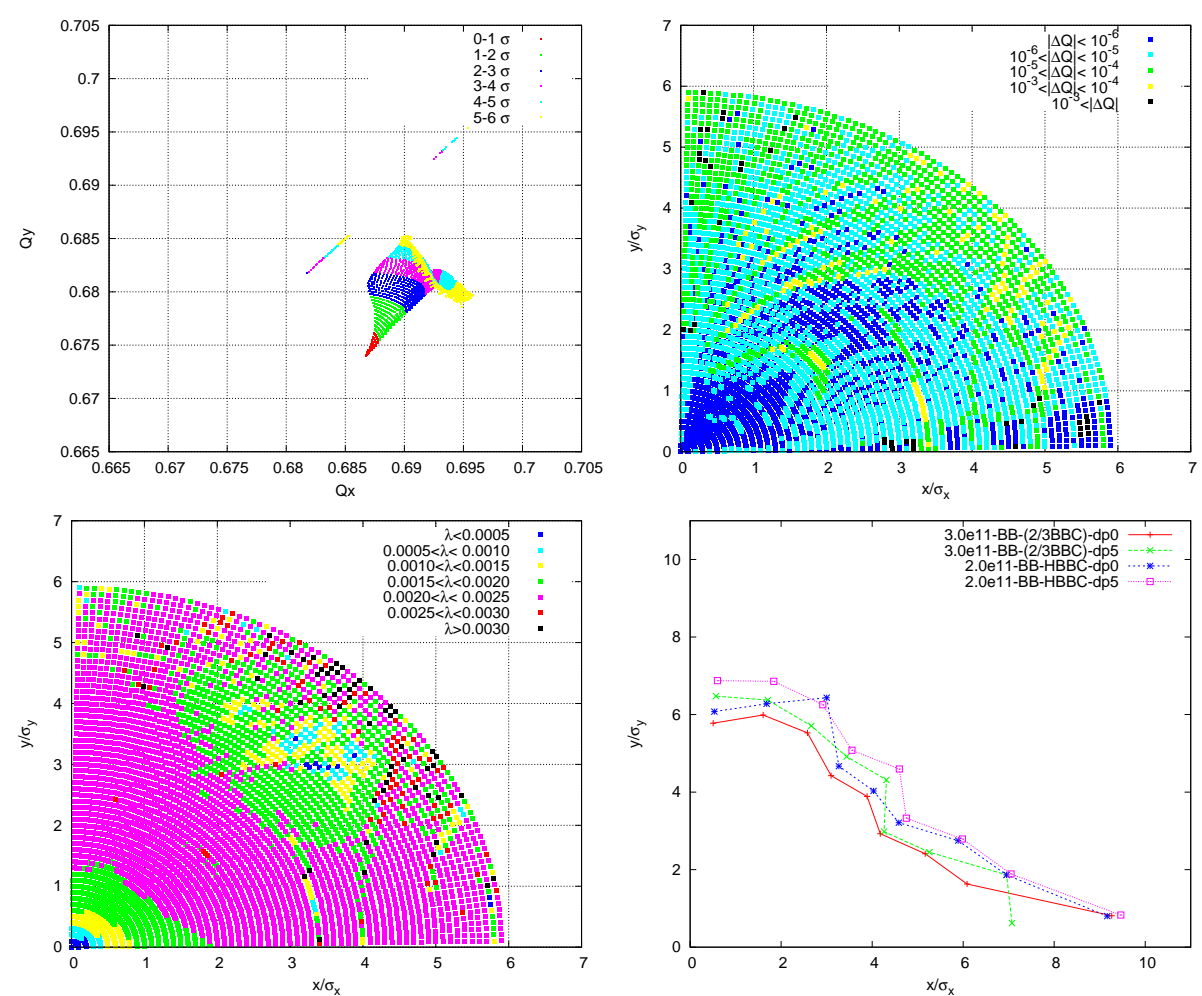

Figure 14: Tune footprint, tune diffusion, Lyapunov exponent, and dynamic apertures with $N_{p}=3.0 \times 10^{11}$ and $2 / 3$ BBC for working point $(28.695,29.685)$.
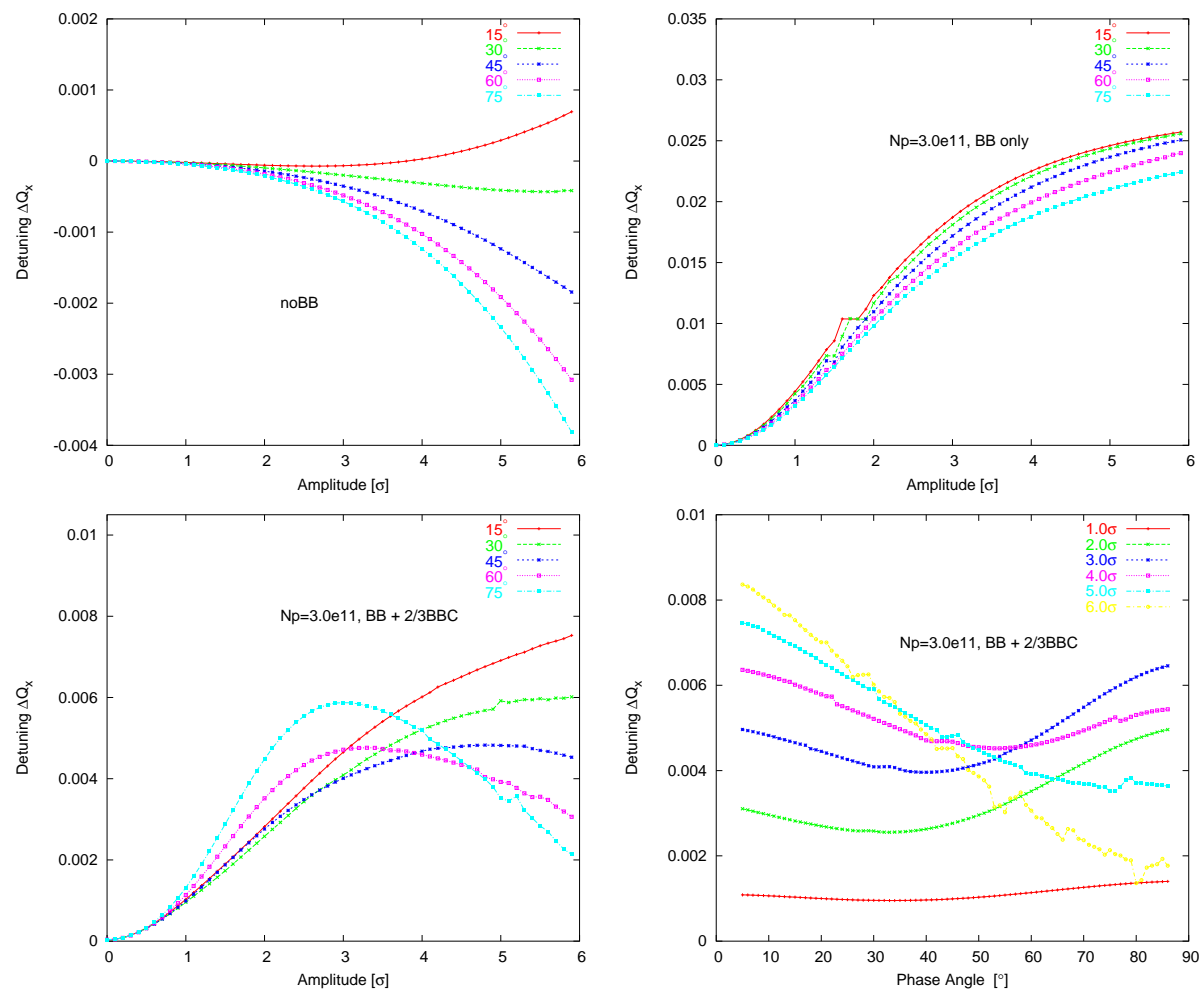

Figure 15: Examples of tune footprint foldings, working point is $(28.685,29.695), N_{p}=3.0 \times 10^{11}$. Topleft: detuning $\Delta Q_{x}$ only from magnetic nonlinearities; Top-right: detuning $\Delta Q_{x}$ only from beam-beam interaction; Bottom-left: radial detuning $\Delta Q_{x}$ with $2 / 3$ head-on beam-beam compensation ; Bottom-right: azimuthal detuning $\Delta Q_{x}$ with $2 / 3$ head-on beam-beam compensation. 